\title{
La gestión de la variación lingüística en vasco y en A Fala del Valle de Jálama: ¿"policéntrica" o "composicional"?
}

\section{The Management of Language Variation in Basque and A Fala: "Pluricentric" or "Compositional"?}

JoAn Costa-Carreras [joan.costa@upf.edu]

Universitat Pompeu Fabra, España

\section{RESUMEN}

El presente trabajo se originó ante la aparente contradicción en la estandarología entre la composicionalidad (Einar Haugen 1968) y el pluricentrismo (Kloss 1967) en la codificación del vasco. No hay tal contradicción: éste se dio cuando la estandarización del euskera estaba en su fase inicial; aquella, cuando la estandarización del vasco ya había avanzado con un elaborado planteamiento de planificación de corpus. Por lo tanto, se habría dado una relación de sucesión. Por otra parte, el análisis que hemos hecho de la fala del Val de Xálima a raíz de la comunicación de Valeš (2019) confirma esta sucesión. Además de deshacer esta posible contradicción, el análisis de diferentes trabajos previos, nos ha permitido identificar diferentes cuestiones relativas al término composicionalidad: la equidad perfecta entre dialectos es una utopía; la composicionalidad comporta un dinamismo en la relación entre estandarización y vernáculos; y la distancia estructural entre los dialectos es un factor que puede condicionar la adopción de la composicionalidad.

\section{Palabras Clave}

A Fala; vasco; estandarología comparativa; composicionalidad; Valle de Jálama; lagarteiru; variación lingüística; pluricentrismo

\footnotetext{
Abstract

The present work originated from the apparent contradiction in the standardization between compositionality (Einar Haugen 1968) and pluricentricity (Kloss 1967) in the codification of Basque. There is no such contradiction: the latter occurred when the standardization of the Basque language was in its initial phase; the former, when the Basque standardization had already advanced with an elaborate corpus planning approach. Therefore, a succession relationship would have occurred. The analysis we have made of "A Fala" of the Val de Xálima following Valeš (2019) confirms this succession. In addition to undoing this possible contradiction, the analysis of the different testimonies has allowed us to identify different issues related to the term compositionality: the perfect equity between dialects is a utopia; the compositionality implies a dynamism in the relationship between standardization and the vernacular varieties; and the structural distance between dialects is a factor that can condition the adoption of compositionality.
} 


\section{KEYWORDS}

A Fala, Basque, comparative standardology, compositionality, Val de Xálima, lagarteiru, language variation, pluricentricity

RECIBIDO 2019-11-12; ACEPTADO 2020-02-27

Es de toda justicia agradecer a nuestra colega Miren Azkárate los comentarios que ha hecho, los cuales han evitado algunas inexactitudes de una primera versión del trabajo. Evidentemente las que puedan subsistir son únicamente responsabilidad nuestra.

\section{Introducción}

El motivo de la presente comunicación tiene que ver con el objetivo general 1 del proyecto "Estandarología comparada entre las lenguas de España (ECLE): la gestión contemporánea de la normatividad", que consiste en proponer un marco teórico epistemológico que contribuya a la consolidación de la estandarología comparada en el contexto sociolingüístico peninsular ibérico y que se concreta en los siguientes objetivos específicos: ${ }^{1}$

a) OE1. Esclarecer el estatus epistemológico y extraepistemológico ${ }^{2}$ de la estandarología comparada, es decir sentar sus bases teóricas y metodológicas.

b) OE2. Proponer una terminología lingüística normalizada y un metadiscurso especializado consensuado para los estudios de estandarología en la Península Ibérica, cuyo producto final esperado es un glosario cuatrilingüe (catalán-gallego-español-vasco).

La exposición va a mostrar cómo ha sido clasificada la relación que la lengua "literaria" o la "estándar” vascas mantienen con sus variedades geográficas: algunos estandarólogos (Polanco 1984; Ruiz, Sanz y Solé 2001; Stillwell \& Hetrovicz 2013) consideran esta relación como un ejemplo de "selección composicional”; otros, como Kloss (1967), ven en ella un ejemplo de "selección policéntrica”:

Polycentric standards will be found where a language is dominant in two or more geographically separated countries (British and American English; Portuguese in Brazil and Portugal) and in speech

1 El autor de este trabajo forma parte del equipo de Estandarología Comparada entre las Lenguas de España (ECLE), que presentó en octubre de 2019 una solicitud de subvención a la Administración española para llevar a cabo en 4 años el proyecto. Forman parte del equipo de investigación para el proyecto, además de los dos investigadores principales (el autor de este trabajo y Miquel Àngel Pradilla, de la Universidad Rovira i Virgili, Tarragona), Carla Amorós-Negre (Universidad de Salamanca; IULATerm), Miren Azkárate (Universidad del País Vasco), Agurtzane Elordui (Universidad del País Vasco), Aina Labèrnia (Universidad Ramon Llull, Barcelona), Henrique Monteagudo (Universidad de Santiago de Compostela) y Elixabete Pérez Gaztelu (Universidad de Deusto). El proyecto se centra en el catalán, el gallego, el español y el vasco, por una razón de oportunidad, pero no está cerrado a colaboraciones con expertos de otras lenguas ibéricas.

2 Por "estatus extraepistemológico" entendemos la situación de la estandarología comparada en el ámbito académico (planes de estudio, doctorados, revistas, etc.) y profesional (profesionales, usuarios, empresas, etc.). 
communities which are still in the beginning stage of their modernization (Albanians, Basques, Kurds, etc.), or where political circumstances have brought about separated developments for two variants of one single language (Roumanian and Moldavian; Serbian and Croatian) (Kloss 1967:31)

Vamos a tratar de explicar esta aparente contradicción. Pero, por su parte, la estandardología vasca (Mitxelena 1981; Garabide Elkartea 2010; Salaburu 2015; Oñederra 2016) no ha usado ninguna de estas denominaciones. Corresponde entonces analizar cómo explica esta estandarología las relaciones entre las variedades geográficas y la lengua estándar: para una primera aproximación a esta tarea tendremos en cuenta el trabajo de Metzeltin (2007). Todo ello se va a comparar con las reflexiones que nos sugirió el trabajo de Miroslav Valeš, sobre "A Fala: Primary data collection for description and documentation" (Valeš 2019): su producción oral y escrita en lagarteiru, ¿fue un caso de cultivo policéntrico o composicional? Se ofrecen finalmente unas conclusiones sobre el conjunto conceptual que se deriva de la presentación y la discusión consiguiente.

\section{El vasco, dado como ejemplo de selección composicional}

Para este trabajo pudimos encontrar testimonios de esta posición en autores que hablaban sobre el caso catalán, unos extranjeros (Stillwell \& Hetrovicz 2013) y otros catalanes (Polanco 1984; y Ruiz, Sanz y Solé 2001).

Stillwell \& Hetrovicz (2013) afirman lo siguiente:

Haugen defines the selection process as one which strives for "minimal variation in form" (1972:107). This requires that either [...] or that a koiné be created, adopting elements of various varieties and combining them into one form which did not previously exist, as in the case of Standard Basque (Hualde \& Zuazo 2007). (Stillwell \& Hetrovicz 2013: 69-70.)

Estos estandarólogos no usan el término composicional para referirse al vasco, pero el proceso que explican para esta lengua, "la creación de una koiné combinando elementos de diversas variedades geográficas que no es ninguna variedad que existiera antes de este proceso", es la definición de "selección composicional". Por lo tanto, atribuyen al vasco la noción de 'composicionalidad' aunque no la denominen de ninguna manera.

En la estandarología catalana, podemos mencionar los trabajos de Polanco (1984) y Ruiz, Sanz y Solé (2001). Polanco (1984: 116:) es el primero de esta estandarología que usa composicional, para explicar el cual dice que "hi ha una sola varietat estàndard creada a partir d'una aportació més o menys igualitària de diferents varietats geogràfiques", y son ejemplos de ella la "koinê grega" y "els moderns dialectes noruecs per Ivan Aasen o el basc unificat". Más adelante, cita a Haugen (1972 [1968]: 265): “There are two rival hypotheses concerning selection": la segunda es "that an $\mathrm{S}$ [tandard] L[anguage] is (or should be) a composite of dialects (the nature of composition being left unspecified). We may call [this thesis] the compositional ${ }^{3}$ thesis. [...]. Both theses have been argued in Scandinavia [...] with inconclusive results [...]". Con esta cita se ve que el creador de esta 
denominación es Einar Haugen en 1968 y que deja sin concretar "la naturaleza de la composición, que queda inespecificada". Esta observación es capital en nuestra exposición.

Por su parte, Ruiz, Sanz y Solé (2001) dan la siguiente información en su Diccionari de sociolingüística:

2 Model composicional o compositiu. [...] amb les aportacions de tots [els dialectes ], encara que un pugui tenir més pes que els altres. L'euskera batua (basc unificat) [en] seria un exemple [...]: no s’identifica amb cap dels dialectes preexistents, sinó que pretén ser-ne la síntesi. (entrada estandardització).

En este punto de la exposición nos parece pertinente retener la afirmación de que un dialecto puede tener más peso que los demás, ya que nos va a permitir explicar que la designación composicional es muy genérica y da cuenta de situaciones muy diversas.

\section{El vasco, dado como ejemplo de selección "policéntrica"}

El único testimonio que hemos encontrado de esta posición es la del creador del término polycentric: Kloss (1967: 31), que afirma como hemos vista en el $\$ 1$ que "polycentric standards will be found [...] in speech communities which are still in the beginning stage of their modernization (Albanians, Basques, Kurds, etc.) [...]”. También nos parece pertinente no perder de vista la caracterización de la comunidad lingüística vasca, en el año 1967, como una comunidad que todavía estaba en el estadio inicial de su modernización, de cara a explicar la aparente contradicción entre la composicionalidad y el pluricentrsimo en la estandarización del vasco.

\section{La base dialectal del estándar según la estandarología vasca}

En esta sección vamos a analizar, como primera aproximación, textos de Koldo Mitxelena (1981), Garabide Elkartea (2010), Pello Salaburu (2015) y Miren Lourdes Oñederra (2016).

Son pertinentes, entre los que hemos tenido tiempo de leer, los siguientes fragmentos de Mitxelena (1981). El primero afirma que "[...] variedades locales y comarcales han tenido que competir [...] con los llamados dialectos literarios ['escritos']” (Mitxelena 1981: 291). El pretérito perfecto compuesto "han tenido" nos sitúa en el pasado, lo que nos permite aducir la fase inicial de modernización del euskera a la que aludía Kloss (1967). En esta fase estaban los “dialectos literarios", que es verosímil suponer que se fueron creando según la necesidad de los escritores que, en su conjunto, no debían de tener una formación gramatical sólida y que sólo pretendían disponer de una variedad para sus objetivos literarios. Ello era una puerta abierta al policentrismo de modelos de lengua literaria. Y la constatación de que "nuestro caso es según toda evidencia análogo al del griego anterior a la época helenística" (Mitxelena 1981: 294), reafirma esta fase inicial de policentrismo antes de una acción de base "composicional".

El dinamismo en las relaciones entre "lengua literaria" o "lengua común" se plasma en la afirmación mitxeleniana de que "se ha llegado [...] a un esbozo de lengua común para el uso escrito 
como primer paso [y lo] que se construya sobre este fundamento podrá servir de punto de referencia para la multiplicidad de dialectos y variedades, de cuya savia [...] se tendrá que nutrir la lengua común” (Mitxelena 1981: 295). Se abre a partir del hito del “esbozo de lengua común” una relación en espiral virtuosa (no un "círculo virtuoso", sino una espiral que evoluciona, que se expande) entre la lengua común (que ha sido fruto de la selección a partir de los dialectos) y los "dialectos y variedades", para los que aquella será el patrón a partir del cual se irán modelando; pero, al mismo tiempo, éstos son el almacén donde aquella podrá acudir para resolver nuevas necesidades expresivas. He aquí, pues, otra situación que cabe bajo el término composicionalidad: los dialectos como "savia", como "almacén", para resolver necesidades expresivas. Y Mitxelena concluye este pasaje diciendo que "[entre los] dialectos vascos [...] las divergencias [...] son desesperadamente pequeñas” (Mitxelena 1981: 295), lo que nos permite introducir la cuestión de los factores que favorecen esta estrategia estandarizadora. Esta estrategia está ligada, a lo que Lamuela (1984) y Bibiloni (1997) llaman “criterio de diasistematicidad". El primero lo define como el

punt de partida [d'una [...] codificació que es proposi d’establir una norma múltiple: la] concepció de la variació $[\ldots]$ com un tot organitzat en el qual existeix una jerarquia de les diverses formes lingüístiques establertes en funció de la mesura en què cada una d’elles explica les seves concurrents [...] el terme que Weinreich [1954] va encunyar per a la dialectologia estructural, l'anomenaré criteri de diasistematicitat (Lamuela 1984: 80).

Por su parte, Bibiloni (1997: 40-41) afirma que la codificación composicional "reclama una codificació que treballi a fons amb el criteri de diasistematicitat". Este criterio está subyaciente en la afirmación de Mitxelena (1981: 309) de que "si tuvo que existir una especie de lengua común como raíz de los dialectos [...] habrá que preguntarse [...] cómo llegó a constituirse [...] por hombres que operan dentro de ciertas condiciones históricas". Esta hipotética lengua común primigenia sólo se podría descubrir aplicando el principio de diasistematicidad. Pero para Mitxelena (1981: 310) la composicionalidad parece ser tan solo una etapa en el proceso de estandarización: "una lengua común supone el sacrificio por extinción de los dialectos en favor de alguno de ellos. [...] la unificación lingüística suele ser resultado (y factor) de cohesión social”.

Es oportuno en este punto recuperar el trabajo de Metzeltin (2007) sobre la codificación de las lenguas románicas, ya que proporciona un marco conceptual en el que vamos a poder situar el vasco - lengua no románica, pero sí elaborada y estandarizada en un contexto románico.

Metzeltin pretende explicar la "creación de una o más lenguas nacionales" en un mismo territorio: "escogiendo una variedad lingüística, dándole unas reglas explícitas y homogeneizándola, los gramáticos del grupo ["nacional”] crean una o más lenguas nacionales con su historia" (Metzeltin 2007: 148). Y continúa:

Todo Estado necesita para la imposición de su organización de uno o más medios de comunicación social eficientes y eficaces, es decir, de lenguas reconocidas de valor y eventualmente también símbolos de identidad nacional. Estas lenguas se crean por selección, homogeneización y reglamentación explícita. Si comparamos la historia de las lenguas nacionales románicas entre sí, podemos observar que su estandarización, es decir, su lenta transformación de variedad dialectal en medio de comunicación oficial generalizado, implica los siguientes momentos: 
- Concienciación (los hablantes se conciencian de la individualidad/alteridad de su lengua por la necesidad de traducciones, glosarios y de una denominación propia).

-Textualización (surgen series textuales como leyes, poesías líricas, crónicas que inauguran tradiciones textuales).

- Codificación (surgen ortografías, ortoepias, gramáticas, diccionarios, retóricas y poéticas que sistematizan explícitamente la lengua).

-Normativización ([...] academias, que a través de una selección de las variantes unifican la lengua y regulan su escritura y declaran que la gramática y el vocabulario así seleccionados son los correctos; sobre todo a partir de este momento se desarrollan la conciencia sociológica de los dialectos [dialectología] [sic] y las actitudes conscientes ante las variedades lingüísticas [etología lingüística] [sic]).

-Oficialización (esta lengua concienciada, textualizada, codificada y normativizada es anclada en una legislación apropiada para que se utilice en la comunicación de los poderes públicos entre ellos y con el público).

- Medialización (los órganos estatales tratan de introducir o imponer la lengua normativizada y oficializada en todas las esferas de dominio público, en particular en las escuelas a través de una enseñanza y manuales apropiados).

-Internacionalización (los órganos estatales tratan de hacer reconocer o imponer su lengua como medio de comunicación internacional).” (Metzeltin 2007: 148)

Si aplicamos al vasco los "momentos" de su "lenta transformación de variedad dialectal en medio de comunicación oficial generalizado", son pertinentes los siguientes comentarios:

a) Concienciación: estando rodeado el vasco al menos desde la romanización por lenguas románicas no siéndolo él, los vascohablantes se debieron de concienciar hace muchos siglos de la individualidad y alteridad de su lengua, no solo por la necesidad de traducciones, glosarios y de una denominación propia, sino también en las interacciones comunicativas cotidianas.

b) Textualización: también en el caso vasco surgen series textuales como leyes, poesías líricas, crónicas que inauguran tradiciones textuales.

c) Codificación: también surgen varias ortografías, ortoepias, gramáticas, diccionarios, retóricas y poéticas, diversas geográficamente, que sistematizan explícitamente la lengua.

d) Normativización: ya en el siglo XX se crea Euskaltzandia (Real Academia de la Lengua Vasca), que a través de una selección de las variantes está unificando la lengua y regulando su escritura y declara que la gramática y el vocabulario así seleccionados son los correctos. A partir de este "momento" se han ido desarrollando la conciencia sociológica de los dialectos y las actitudes conscientes ante las variedades lingüísticas, proceso aun en curso en el siglo XXI.

e) Oficialización. a finales del siglo XX esta lengua concienciada, textualizada, codificada y normativizada fue anclada en una legislación apropiada para que se utilice en la comunicación de los poderes públicos entre ellos y con el público.

f) Medialización: desde finales del siglo XX, los órganos autonómicos han tratado de introducir o imponer la lengua normativizada y oficializada en todas las esferas de dominio público, en particular en las escuelas a través de una enseñanza y manuales apropiados.

g) Internacionalización: los órganos autonómicos no han tratado de hacer reconocer o imponer su lengua como medio de comunicación internacional. 
Para valorar el grado de "nacionalización” (de compleción como "lengua nacional”) del vasco en el ámbito de la Comunidad Autónoma del País Vasco del Estado español, podemos compararlo con el del español, que en principio ha cumplido y cumple en su grado máximo esta "nacionalización”. Para empezar, el vasco igual como las otras lenguas del Estado español que no son el español no ha experimentado la internacionalización en el sentido de Metzeltin, si bien es cierto que se puede aprender fuera del País Vasco, lo que no deja de ser un tipo de "internacionalización”. Se puede decir que el vasco ha cumplido y cumple satisfactoriamente los "momentos" de concienciación, textualización, codificación y normativización. Donde ha habido y hay más problemas es en la oficialización y en la medialización. En cuanto a la primera, hay vacíos importantes en el cumplimiento de la legislación en la que el euskera está "anclado", a pesar de ser "apropiada para que se utilice en la comunicación de los poderes públicos entre ellos y con el público". Por lo que respecta a la medialización, dada la presencia del español y del inglés, la introducción o imposición de la lengua normativizada y oficializada en todas las esferas de dominio público es difícil en muchas de ellas (la justicia, el ocio audiovisual), a pesar del gran esfuerzo hecho por la Administración autonómica. En cuanto a la escuela, el llamado modelo D (enseñanza en euskera como lengua vehicular, con asignatura de Lengua Castellana y Literatura) escolariza a casi el 60\% del alumnado preuniversitario, salvo en la Formación Profesional, donde existe una laguna importante. Ello significa un "anclaje" importante pero no total del euskera en la enseñanza, por lo menos en la Comunidad Autónoma del País Vasco.

¿Qué aporta el planteamiento de Metzeltin (2007) a la discusión sobre el policentrismo o la composicionalidad del vasco? Siguiendo a este romanista suizo, se puede decir que el primero se dio en las fases de concienciación, textualización y codificación cuando la intervención sobre la lengua o alguno de sus dialectos era llevada a cabo por personas cuya necesidad inmediata y única era disponer de un código para satisfacer sus carencias de comunicación escrita, sin ningún planteamiento político de dotar a la "nación" de una variedad única. La ausencia de este planteamiento político unificador es el factor que explica que cada redactor o escritor o grupo de redactores o escritores buscara codificar su propia variedad sin ir más allá. En cambio, la composicionalidad se plantea en la normativización y medialización, que se llevaron y se están llevando a cabo en un contexto cultural, social, ideológico y político mucho más respetuoso con la diversidad, no sólo entre los destinatarios de la intervención sobre la lengua sino entre los propios normativizadores y medializadores.

No se puede descartar que en un futuro pueda resurgir el policentrismo, si se dan actitudes de revalorización de los rasgos estándares propios de alguna zona, como ha pasado en el mundo panhispánico, por ejemplo.

Por otra parte, Metzeltin (2007: 150) cita a Haugen (1959: "Planning in Modern Norway"), lo que nos permite ampliar nuestra reflexión sobre el caso del vasco. Completamos este análisis con la comparación del modelo de Metzeltin con el de Haugen (1983) y con la perspectiva de las funciones sociales que según Garvin (1992) cumple una variedad estándar bien establecida:

a) En la concienciación, operan las funciones sociales garvinianas de aglutinación interna (conciencia de hablar una comunidad la misma lengua) y de diferenciación externa (conciencia de que esta lengua es única y diferente de las demás).

b) La textualización puede ser definida en términos haugenianos como una "elaboración funcional" discrecional a medida que había que resolver necesidades expresivas. 
c) Las diferentes "codificaciones" eran también codificaciones discrecionales y particularistas, limitadas a la propia variedad de los redactores o escritores.

d) En la normativización, oficial, ya se hace una "selección" de una o unas variedades geográficas y se aspira a que esta variedad o variedades seleccionadas y codificadas operen, en términos de Garvin (1992), como "marco de referencia” de la adecuación de formalidad: qué es apropiado y qué no en según qué circunstancias.

e) La oficialización es una decisión de planificación lingüística de estatus (Kloss 1969: 81) y conlleva la difusión de la variedad o variedades seleccionadas y codificadas, y aspira a dotarlas de prestigio otra de las funciones identificadas por Garvin (1992) como propias de una variedad estándar. También tendría que conllevar su carácter de requisito para trabajar al menos en la Administración pública (la llamada función discriminante), ésta propuesta por Lamuela (1994).

f) La medialización consiste en la difusión haugeniana.

g) La internacionalización metzeltiniana no forma parte estrictamente de un proceso de estandarización: ésta puede existir sin aquella. Si se toma este "momento" como la capacidad de las variedades codificadas de servir a sus hablantes para recibir cualquier tipo de información desde cualquier parte del mundo y para referirse a cualquier tema de cualquier ámbito, entonces estas variedades ejercen lo que Garvin (1992) llama función de participación.

h) Quizás porque se detiene a principios del siglo XX, el modelo de Metzeltin no prevé la cuarta fase del modelo haugeniano: la elaboración funcional deliberada y sistemática, sin la cual actualmente las lenguas no pueden responder a las constantes y acuciantes necesidades de denominación de las novedades constantes en la sociedad.

¿Qué funciones sociales cumple el actual Euskara Batua? Cumple, sin lugar a dudas, como ya hemos dicho antes por la distancia estructural entre el vasco y sus vecinas románicas, las funciones de aglutinación interna (identificación entre los “euskaldunes") y diferenciación externa (el euskera es una lengua diferente de las demás). En cuanto a la función de "marco de referencia" la cumple para todas las personas escolarizadas en euskera, pero con una importante presión del marco de referencia del español, dado que los euskaldunes también conocen y usan esta lengua.

A partir de ahí, hay un cumplimiento incompleto de las otras funciones. La oficialización incompleta en todos los ámbitos sociales comporta un prestigio parcial. Sin un doblaje sistemático de los productos audiovisuales al euskera, no puede competir con el español en la función de participación. Y si no es exigida sistemáticamente, aunque sea con niveles de competencia mínimos, en el mundo laboral tampoco ejerce la función discriminante.

La segunda fuente sobre estandarología en la que nos vamos a fijar es Garabide Elkartea (2010). En primer lugar afirma que "the point of departure [of Mitxelena's works] was linked to the basic idea that it was not the differences between spoken varieties that required attention, but the common core" y que "Mitxelena based his proposal on the central dialect" (Garabide Elkartea 2010: 32). Esta afirmación daría a entender que Mitxelena debido a la diversidad sobre todo en morfología no debía de prever un recurso sistemático a la composicionalidad, lo que nos lleva a otro elemento a tener en cuenta: el grado o la sistematicidad de la composicionalidad de una codificación. Graduación discrecional que se confirma con la afirmación de que "notwithstanding the importance attached to the central dialects, if the peripheral dialects coincided on something, that 
should be preferred." (Garabide Elkartea 2010: 33). Y participa de la relación en espiral virtuosa entre el estándar y las variedades geográficas: "Nowadays Euskaltzaindia acknowledges dialectal vocabulary as an asset to be maintained and exploited" (Garabide Elkartea 2010: 50).

Por su parte, Salaburu (2015: 10) se pregunta “why was Euskaltzandia formed?” y contesta: "In order to] [...] determine a standard or unified Basque: to specify "a Basque model that grows up from the blood of all the dialects [...]". Si antes para Mitxelena los dialectos eran la "savia", ahora son la "sangre", no sólo porque lo fueron al constituirse la lengua común, sino porque van a permitir transfusiones discrecionales cuando ésta las necesite.

Finalmente, exponemos lo que Oñederra (2016: 346), especialista en ortoepia, afirma:

[T] he challenge for the linguist would be to find a way to make Standard Basque phonologically compatible with the dialects. A necessary condition for that to be feasible is that all the participants in the standardisation process (rulers, teachers and other speakers) understand that an oral standard will always be more variegated than a written one. That is to say, different dialectal colourings will give rise to several standard pronunciations (Oñederra 2016: 346).

Este fragmento nos permite introducir la cuestión de si la composicionalidad es más fácil según el nivel de lengua que se codifique (fonética, morfología, léxico, sintaxis o textualidad) o según si trata de la codificación ortográfica.

Para la ortoepia, Oñederra subraya que "the main dialectal characteristics to be incorporated into those oral standards should be very carefully chosen” (Oñederra 2016 347).

\section{La comunicación de Valeš (2019): ¿un caso de cultivo composicional o policéntrica?}

En esta sección vamos a presentar las reflexiones que nos surgieron a medida que íbamos escuchando la interesantísima comunicación que Miroslav Valeš dio en "lagarteiru” en Lisboa en julio de 2019 (Valeš 2019). ${ }^{4}$

Valeš declaró que daba su presentación en una de las tres "falas" de la fala: el lagarteiru. La presentación que hizo explicó que en intervenciones como el establecimiento de una ortografía (Valeš 2017) hay una cierta base policéntrica (si las decisiones se tomen por separado en cada comunidad) o composicional (si las toma un organismo único): se respetan los rasgos más significativos de cada una de las falas, se prevén dobletes o tripletes gráficos y los hablantes tienen voz y voto.

La pregunta que nos hacemos y que Valeš no nos ha podido responder es por qué escogió el lagarteiru, al menos para la expresión oral. Por lo tanto, cabe suponer que la pronunciación, la morfología y el vocabulario general debían de ser lagarteiranas. En cuanto a la sintaxis, como es general en todas las lenguas, las diferencias deben de ser menores. Pero la producción oral que Valeš ejecutó era una exposición especializada: ¿de dónde sacó la terminología especializada? ¿Recurrió sistemáticamente al préstamo del portugués o del español?

4 Lamentablemente, no nos ha sido posible contactar con él para contrastar nuestras especulaciones. 
En cuanto a la grafía usada para las diapositivas, cabe suponer que usó la elaborada en Valeš (2017), que "intenta respetal [...] as tradición singulais dus tres lugaris, sugirindu «grafías equivalentis» pa que cada un dus falantis se poda sentil indentificau con a ortografía” (Valeš 2017: "Prólugu da versión actualizá (2017)”). Hay que deducir que si escogió el lagarteiru para su exposición oral, debió de seleccionar, cuando era necesario, las "grafías equivalentes" correspondientes al lagarteiru. Desde este punto de vista, el orador escogió una perspectiva policéntrica de elaboración.

La perspectiva hasta aquí de nuestras reflexiones ha sido desde la perspectiva del emisor: ¿cómo se hubieran sentido los hablantes de la fala escuchando dicha presentación? Obviamente, habría que tener en cuenta que fue una exposición teórica especializada. Pero justamente eso parece formar parte del problema: como por ejemplo descubrió Jaffe (2005) para el corso, los hablantes de lenguas minoritarias y minorizadas (o nunca "mayorizadas", desarrolladas, cultivadas), no se identifican (e incluso rechazan) las variedades elaboradas de su propia lengua. La pregunta, pues, es: ¿se identificarían los hablantes de las tres falas con versiones elaboradas de sus vernáculos? ¿Todos estos hablantes entenderían cualquier variedad elaborada de lagarteiru, mañegu o valverdeñu? La respuesta a esta pregunta tiene que ser afirmativa, ya que estamos hablando de tres variedades de una lengua con 3.500 hablantes, cuya mutua comprensión es del 100\%.

\section{Comparación de la estandarización del vasco con la elaboración realizada por Valeš (2019) sobre A Fala}

En la sección 4 hemos llegado a unas conclusiones sobre el vasco a la luz de los trabajos de Metzeltin (2007), Haugen (1983), Garvin (1992) y Lamuela (1994). Si trasladamos estas perspectivas a la reflexión hecha sobre la presentación de Valeš (2019), podemos decir lo siguiente.

Tal como decíamos allí para el euskera, la fala no ha experimentado la internacionalización en el sentido de Metzeltin. En cuanto a aprenderlo fuera del Val de Xálima, no debe de ser fácil. Se puede decir que la fala ha cumplido y cumple satisfactoriamente los "momentos" metzeltinianos de concienciación, textualización y codificación, aunque haya más de una. Donde ha habido y hay más problemas es en la oficialización, la normativización y en la medialización. En primer lugar, la fala tiene un reconocimiento mínimo como "Bien de Interés Cultural” por la Junta de Extremadura, lejos de la cooficialidad. En segundo lugar, no se ha creado ninguna academia oficial que de manera centralizada trabaje en una ortografía, una gramática y un diccionario. Finalmente, tiene una cierta difusión, pero, fruto de la falta de cooficialidad, no se mueve del ámbito privado, especialmente en las relaciones sociales primarias (familia y amistades): no se enseña en las escuelas.

¿Qué aporta el planteamiento de Metzeltin (2007) a la discusión sobre el policentrismo o la composicionalidad de la fala? El primero se dio en la fase de concienciación y se está dando en los "momentos" actuales de textualización y codificación: la intervención sobre la fala o alguna de las falas es llevada a cabo por personas cuya necesidad es disponer de un código para satisfacer sus carencias de comunicación escrita, según parece sin ningún planteamiento político de dotar al Val de Xálima de una variedad única. La ausencia de este planteamiento político unificador es el factor que explica que cada grupo de redactores o escritores busque codificar su propia variedad sin ir más allá. En cambio, queda por ver si la composicionalidad se plantea en la incipiente normativización y medialización, que se están llevando a cabo en un contexto cultural, social, ideológico y 
político mucho más respetuoso con la diversidad, no sólo entre los destinatarios de la intervención sobre la lengua sino entre los propios normativizadores y medializadores.

En cuanto a las aportaciones que se pueden extraer de los análisis de Haugen (1983) y de las funciones sociales que según Garvin (1992) y Lamuela (1994) cumple una variedad estándar bien establecida, podemos decir en relación con la fala:

a) En la concienciación, operan las funciones sociales garvinianas de aglutinación interna y de diferenciación externa.

b) La textualización puede ser definida en términos haugenianos como una "elaboración funcional" discrecional a medida que había que resolver necesidades expresivas.

c) Las diferentes "codificaciones" son también codificaciones discrecionales y particularistas, limitadas a la propia variedad de los redactores.

d) La normativización, oficial, de la fala es, como máximo, incipiente. Por lo tanto, habrá que ver si se "selecciona" una o unas variedades geográficas y se aspira a que esta variedad o variedades seleccionadas y codificadas operen, en términos de Garvin (1992), como "marco de referencia" de la adecuación de formalidad.

e) No ha habido oficialización de la fala. Queda pendiente, pues, la consecución de prestigio. y, aún más lejos, su posible carácter de requisito para trabajar al menos en la Administración pública (la llamada función discriminante).

f) La medialización, la difusión haugeniana, apenas supera el ámbito privado.

g) Desconocemos si la fala tiene la capacidad de servir a sus hablantes para recibir cualquier tipo de información desde cualquier parte del mundo y para referirse a cualquier tema de cualquier ámbito, lo que Garvin llama función de participación. La comunicación de Valeš (2019) es un paso en esta dirección.

h) En cuanto a la elaboración funcional deliberada y sistemática, sin la cual actualmente las lenguas no pueden responder a las urgentes y acuciantes necesidades de denominación de las novedades constantes en la sociedad, es verosímil que esté muy poco desarrollada en la fala.

¿Qué funciones sociales cumple la fala? Cumple las funciones de aglutinación interna (identificación entre los "falantis") y diferenciación externa (la fala es una lengua diferente de las demás). A partir de ahí, hay un cumplimiento incompleto de las otras funciones. Si no hay una norma, es imposible su difusión en el sistema de la enseñanza, y no ejercerá la función de "marco de referencia" para todos los "falantis". La falta de oficialización comporta una ausencia de prestigio. Sin acceso a las producciones audiovisuales o traducciones de obras literarias, la fala tampoco cumple la función de participación. Y si no es exigida en el mundo laboral tampoco ejerce la función discriminante.

Si comparamos estas observaciones sobre la fala con las hechas sobre el vasco, se ve que aquella está en la fase metzeltiniana de "normativización" y aún no exactamente, con una medialización reducida y sin oficialización ni internacionalización. Ello explicaría que se dé la típica situación de policentrismo de la "codificación" en términos de Metzeltin (2007), de la cual cabe suponer que la comunicación de Valeš (2019) sería un exponente. 


\section{Conclusiones}

El presente trabajo se originó ante la aparente contradicción en la estandarología entre la composicionalidad (término creado por Einar Haugen en 1968 para no usarlo jamás) o el policentrismo (término creado por Kloss en 1967) de la selección de la variedad a codificar en la lengua vasca. No hay tal contradicción si se tiene en cuenta las fechas de cada una de las definiciones. Kloss escribe cuando la estandarización del euskera está en su fase inicial, y por lo tanto empieza a trabajar en una situación de partida en la que hay, por una parte, varios dialectos literarios, creados por escritores para obras de ficción, y las variedades vernáculas, por otra. Los “composicionalistas" escriben mucho más tarde, a partir de 1984, cuando la estandarización del vasco ya ha avanzado con un elaborado planteamiento de planificación de corpus, no con el fin de disponer de una variedad sólo para la ficción, sino con el objetivo de establecer una variedad de uso común. Por lo tanto, se habría dado una relación de sucesión entre un proceso largo, no sistemático, ad hoc, discrecional, de codificación, en el sentido de Metzeltin (2007), que habría llevado a un "policentrismo de hecho", no buscado, sino simplemente hallado. A esta fase, no coordinada, no centralizada, no llevada a cabo por una institución codificadora, le habría sucedido una fase de estandarización sistemática con un tratamiento global de la diversidad lingüística, incluidos los "dialectos literarios" fruto de dicho policentrismo. El análisis que hemos hecho de la fala a raíz de la comunicación de Valeš (2019) confirma esta sucesión entre policentrismo y composicionalidad.

Además de deshacer esta posible contradicción, el análisis de los diferentes testimonios nos ha permitido identificar diferentes cuestiones relativas al término composicionalidad:

a) La equidad perfecta (Costa Carreras, en prensa) en las fases haugenianas de la selección y la codificación parece una utopía: siempre habrá un o unos dialectos que tengan más o menos peso que los demás.

b) En la estandarización del vasco y de la fala se cumple la sucesión que hemos observado entre policentrismo y composicionalidad: el primero acostumbra a ser característico de los "momentos" de concienciación, textualización y codificación; el segundo se plantea en la normativización, en la medialización y en la internacionalización (si se entiende por ejemplo como ser lengua extranjera de aprendizaje). Estos momentos se han dado y se dan en vasco en un contexto de máximo respeto hacia la diversidad.

c) La composicionalidad presenta un cierto dinamismo: los dialectos no sólo pueden ser la base para conseguir una primera versión de la variedad común, sino que, incluso cuando ésta pueda ser ya percibida como una variedad autónoma de los vernáculos, los dialectos son usados como almacén de recursos para satisfacer nuevas necesidades expresivas. Este dinamismo también tiene que ver con la posibilidad de que esta estrategia sea abandonada en un momento determinado. Para Mitxelena (1981:310), por ejemplo, la composicionalidad parece ser tan sólo una etapa en el proceso de estandarización: "una lengua común supone el sacrificio por extinción de los dialectos en favor de alguno de ellos. [...] la unificación lingüística suele ser resultado (y factor) de cohesión social".

d) La distancia estructural (real o percibida) y el grado de intercomprensión entre los dialectos es un factor que puede condicionar la adopción de la composicionalidad. Esta estrategia está ligada, a lo que Lamuela (1984) llama “criterio de diasistematicidad", que define como el "punt 
de partida [d’una [...] codificació que es proposi d’establir una norma múltiple: la] concepció de la variació [...] com un tot organitzat en el qual existeix una jerarquia de les diverses formes lingüístiques establertes en funció de la mesura en què cada una d’elles explica les seves concurrents [...] el terme que Weinreich [1954] va encunyar per a la dialectologia estructural, l'anomenaré criteri de diasistematicitat" (Lamuela 1984: 80).

e) Por su parte, Bibiloni (1997: 40-41) afirma que la codificación composicional "reclama una codificació que treballi a fons amb el criteri de diasistematicitat". Este criterio está subyacente en la afirmación de Mitxelena (1981: 309) de que "si tuvo que existir una especie de lengua común como raíz de los dialectos [...] habrá que preguntarse [...] cómo llegó a constituirse [...] por hombres que operan dentro de ciertas condiciones históricas".

\section{Referencias bibliográficas}

Bibiloni, G. (1997). Llengua estàndard i variació lingüística. València: Tres i Quatre.

Costa Carreras, J. (en prensa). La relació entre l'estàndard i els dialectes en català: una visió des de l’equitat. In E. Boix \& M $\mathrm{M}^{\mathrm{a}}$ P. Perea (Eds.), Llengua i dialectes: esperances per al català, gallec i basc. Barcelona: Publicacions i Edicions de la Universitat de Barcelona.

Garabide Elkartea (Barandiaran, A., coord.). (2010). Language standardisation. Basque recovery II. Eskoriatza: Garabide Elkartea.

Garvin, P. (1992). La langue standard: Concepts et processus. In II Congrés Internacional de la LlenguaCatalana. Ȧrea 3: Lingüística social (pp.503-514). Palma:Universitat deles Illes Balears.

Haugen, E. (1959). Planning in Modern Norway. Anthropological Linguistics, 1, 3, 8-21.

- (1972 [1968]). The Scandinavian languages as cultural artifacts. In The ecology of Language. Essays by Einar Haugen. Selected and introduced by Anwar S. Dil (pp. 265-286). Stanford: Stanford University Press.

(1983). The implementation of corpus planning: theory and practice. In J. Cobarrubias \& J. A. Fishman (Eds.), Progress in Language Planning. International Perspectives (pp. 269-289). Amsterdam-New York-Berlin: Mouton.

Jaffe, A. (2005), Corse radiophonique élaboré et évaluation populaire: Perspectives corses sur le purisme linguistique, Langage et Société, 112, 2, 79-97.

Hualde, J. I.; \& Zuazo, K. (2007) The standardization of the Basque language. Language Problems and Language Planning, 31, 2, 143-168.

Kloss, H. (1967). 'Abstand Languages' and 'Ausbau Languages'. Anthropological Linguistics, 9, 7, 29-41.

. (1969). Research Possibilities on Group Bilingualism: A Report. [Montreal:] International Centre for Research on Bilingualism, Quebec (Canada).

Lamuela, X. (1984). Fixació i funcionament de la gramàtica normativa en el procés d'estandardització de la llengua catalana. In Ma T. Cabré et al. (Ed.), Problemàtica de la normativa del català. Actes de les Primeres Jornades d'Estudi de la Llengua Normativa. Departament de Llengua Catalana de la Universitat de Barcelona, 30 de setembre i 1 d’octubre de 1983 (pp. 65-92). Barcelona: Publicacions de l'Abadia de Montserrat.

. (1994). Estandardització i establiment de llengües. Barcelona: Edicions 62. 
Metzeltin, M. (2007). Del Renacimiento a la actualidad (I). Procesos de codificación de las lenguas románicas. In J. E. Gargallo Gil \& Ma R. Bastardas (Eds.), Manual de lingüística románica (pp. 147-198). Barcelona: Ariel.

Mitxelena, L. (1981). Lengua común y dialectos vascos. ASJU, 15, 289-313. <http://www.ehu.es)ojs/index. php/asju>

Oñederra, M. L. (2016). The Standardisation of Pronunciation: Basque Today, between Maintenance and Variation. In I. Tieken-Boon van Ostade, \& C. Percy (Eds.), Prescription and Tradition in Language Establishing Standards across Time and Space (pp. 342-354). Bristol: Multilingual Matters.

Polanco Lluís, B. (1984). La normativa al país Valencià. Problemàtica i perspectives. In $\mathrm{M}^{\mathrm{a}} \mathrm{T}$. Cabré et al. (Ed.), Problemàtica de la normativa del català. Actes de les Primeres Jornades d'Estudi de la Llengua Normativa. Departament de Llengua Catalana de la Universitat de Barcelona, 30 de setembre i 1 d'octubre de 1983 (pp. 107-146). Barcelona: Publicacions de l'Abadia de Montserrat.

Ruiz, F.; Sanz, R.; \& Solé, J. (2001). Diccionari de sociolingüística. Barcelona: Enciclopèdia Catalana.

Salaburu, P. (2015). Writing words. The unique case of the standardization of Basque. Reno: University of Nevada.

Stillwell, K.; \& Hetrovicz, L. (2013). The standardizations of Catalan: Latin to present day" In Studies in the Linguistic Sciences: Illinois Working Papers, 68-86.

Valeš, M. (coord.). (2017). Ortografía de A Fala : valverdeñu. S. 1.: Asociación Cultural A Nosa Fala.

Valeš, M. (2019). A Fala: Primary data collection for description and documentation. In Línguas e variedades linguísticas ameaçadas na Península Ibérica (LAPI 2019), 4-5 de Julho 2019, Centro de Inovação da Mouraria / Mouraria Creative Hub, Lisboa (Portugal).

Weinreich, U. (1954). Is a Structural Dialectology Possible? Word, 10, 2-3, 388-400. doi: 10.1080/00437956.1954.11659535. 